\title{
Infliximab in the Treatment of Anti-CTLA4 Antibody (Ipilimumab) Induced Immune-Related Colitis
}

\author{
David R. Minor, ${ }^{1}$ Kevin Chin, ${ }^{2}$ and Mohammed Kashani-Sabet ${ }^{3}$
}

\begin{abstract}
The anti-CTLA4 antibody, ipilimumab, has shown clinical activity against melanoma. Diarrhea due to immunerelated colitis is the most frequent serious toxicity and, if untreated, may lead to intestinal perforation. Diarrhea treatment guidelines were developed based on clinical experience in over 2000 patients treated with ipilimumab, and these safety guidelines recommend systemic steroids as the first choice for the treatment of severe diarrhea. In this article, we present an alternative approach to the control of immune-related colitis by using the antitumor necrosis factor antibody, infliximab. Patients with metastatic melanoma received ipilimumab $10 \mathrm{mg} / \mathrm{kg}$ every 3 weeks for 4 doses, then every 3 months. Those who developed grade 2 diarrhea were treated with infliximab $5 \mathrm{mg} / \mathrm{kg}$ weeks 0 and 2 with mesalamine and loperamide. Steroids were given only for refractory cases requiring hospitalization. Of the first 3 cases of ipilimumab-induced diarrhea, 2 proved refractory and required hospitalization, but 1 recovered quickly without systemic steroids. We then added hydrocortisone enemas daily to the above regimen, and the next 3 patients recovered from grade 2 ipilimumab-induced colitis without difficulty. Treatment with infliximab, mesalamine, and hydrocortisone enemas may produce a rapid improvement in ipilimumab-induced colitis and avoid the administration of systemic steroids.
\end{abstract}

Key words: infliximab, ipilimumab, melanoma, colitis, immune-related, anti-CTLA4

\section{Introduction}

T he discovery of the importance of cytotoxic T-lymphocyte antigen 4 in the regulation of T-lymphocyte activity has led to the introduction of anticytotoxic T-lymphocyte- 4 (CTLA-4) antibodies as immunotherapeutic agents. Extensive clinical trials of two antibodies, ipilimumab and tremelimumab, have been reported in several different cancers, with the demonstration of important clinical activity for ipilimumab in the treatment of melanoma, where durable complete responses have been observed. ${ }^{1-3}$ The safety profile of both of these antibodies is characterized by immune-related adverse events (irAEs) affecting the skin, gastrointestinal (GI) tract, liver, and other organs. These irAEs are usually mild or moderate in severity and are either reversible or clinically manageable. Diarrhea is the most frequent GI irAE and, if untreated, may lead to serious complications, such as intestinal perforation, that could lead to death. ${ }^{4,5}$ Grade 2 or worse diarrhea occurs in about 35\% of patients treated with ipilimumab. ${ }^{6}$ Colitis appears to be the dose-limiting toxicity for both anti-CTLA- 4 antibodies and has been reported to recur when ipilimumab therapy is followed by high-dose interleukin (IL)-2. ${ }^{7}$ Diarrhea treatment guidelines were developed in cooperation with GI experts and have been successfully implemented into ipilimumab clinical trials. Based on clinical experience in over 2000 patients treated with ipilimumab, these guidelines recommend systemic steroids as a first choice for the treatment of severe GI irAEs. In rare cases of steroidrefractory diarrhea or colitis, alternative therapies are needed.

Some researchers ${ }^{5}$ feel there is a correlation between the observation of an immune-related toxicity and a clinical antitumor response. For safety reasons, present guidelines ${ }^{8}$ call for permanent discontinuation of therapy with these agents after the occurrence of grade 3 diarrhea or colitis even in patients who demonstrate a complete response to therapy. This may decrease the duration of remissions, as current

\footnotetext{
${ }^{1}$ California Pacific Medical Center, San Francisco, CA.

${ }^{2}$ Bristol-Meyers Squibb, Wallingford, CT.

${ }^{3}$ The Melanoma Center, University of California, San Francisco, CA.
}

Address for correspondence: David R. Minor, California Pacific Medical Center, San Francisco Oncology Associates; 2100 Webster Street, Suite 326, San Francisco, CA 94115; Tel.: 415-855-8600; Fax: 415-855-8680 
protocols for both agents recommend maintenance therapy, which is felt to be useful in prolonging remissions.

We entered 30 patients into a series of clinical trials of ipilimumab for the treatment of melanoma between January 2005 and December 2007. This included 21 patients on Bristol-Myers Squibb (Wallingford, CT) protocols 007, 008, and 045, which all administered ipilimumab at a dose of $10 \mathrm{mg} / \mathrm{kg}$ every 3 weeks for 4 doses, and 9 patients on Medarex (Princeton, NJ) protocol MDX010-20, of whom 80\% were randomized to ipilimumab $3 \mathrm{mg} / \mathrm{kg}$ for 4 doses. Six (6) of those 30 patients developed colitis and were treated following the recommended guidelines by using systemic steroids first. As outpatients, 1 received budesonide $9 \mathrm{mg}$ daily and 2 received prednisone or its equivalent at $40 \mathrm{mg}$ daily prior to hospitalization. Three (3) patients required urgent hospitalization without outpatient steroids for grade 3 colitis with severe dehydration or bleeding. After hospitalization, the 6 patients' colitis was treated with high-dose systemic steroids, usually methylprednisolone in a dose of over $100 \mathrm{mg}$ daily, but in no case did we observe a significant clinical response to the initial week of high-dose steroids, even when combined with mesalamine. For patients who failed to respond to steroids, we administered the antitumor necrosis factor antibody, infliximab, which is approved by the U.S. Food and Drug Administration for Crohn's disease and ulcerative colitis. Infliximab was given in 2 doses of $5 \mathrm{mg} / \mathrm{kg}$ two weeks apart, according to the approved dosage guidelines for Crohn's disease or ulcerative colitis. On several occasions, we observed a dramatic improvement within 48 hours in the patient's clinical condition after the administration of infliximab. No patients developed intestinal perforation, but these 6 patients required prolonged hospitalizations for an average of 21 days. One (1) patient developed candidemia as a preterminal complication, perhaps, in part, due to the prolonged administration of high-dose steroids.

Although clinical antitumor responses have been observed in patients treated with high-dose steroids for immunerelated toxicities from ipilimumab, steroids have the potential to inhibit T-lymphocyte function and thus may impact antitumor cytotoxicity and, subsequently, limit clinical benefit from ipilimumab treatment. In addition, there have been reports of opportunistic infections in patients treated with prolonged high-dose steroids for ipilimumab-induced colitis. ${ }^{9}$ Given the dramatic clinical responses to infliximab we observed in patients with severe steroid refractory colitis, we explored an alternative approach to treating grade 2 ipilimumab-induced colitis by using infliximab without systemic steroids. The mechanism of action of infliximab is multifold, ${ }^{10,11}$ but blocking the ability of tumor necrosis factor to recruit neutrophils to the site of inflammation in the colon is felt to be important, and blocking this inflammatory pathway should be unlikely to affect the antitumor activity of ipilimumab. Histologic studies of ipilimumab-induced immune-related colitis demonstrate the colonic mucosa infiltrated with both lymphocytes and neutophils, similar to idiopathic ulcerative colitis. ${ }^{12,13}$ Although there is an increased incidence of reactivation of tuberculosis in patients receiving infliximab, large studies ${ }^{14}$ showed no increase in other infections or the incidence of melanoma or other malignancies. ${ }^{15}$ Recent controlled studies of the treatment of Crohn's disease ${ }^{16,17}$ and ulcerative colitis ${ }^{18}$ favor a "top-down" approach to treatment, with the early institution of infliximab while reserving systemic steroids for especially refractory cases. ${ }^{19}$ We felt this approach, using the early administration of infliximab without systemic steroids, might represent an alternative treatment modality for some patients with severe immune-related colitis. In this case study, we report our own clinical experience in 6 cases where this alternative approach was used in the treatment of ipilimumab-induced colitis.

\section{Methods}

Between December 2007 and June 2008, a total of 18 patients at California Pacific Medical Center (San Francisco, CA) were entered onto the expanded access clinical trial of ipilimumab as second-line therapy for patients with metastatic melanoma, and 2 patients were treated on BristolMeyers Squibb study 025, a study of maintenance ipilimumab in patients who had experienced responses in prior ipilimumab protocols. The trials were approved by the Institutional Review Board for the Protection of Human Subjects at California Pacific Medical Center, and all patients gave written informed consent. All patients had metastatic melanoma progressive after prior chemotherapy or biochemotherapy and received single-agent therapy with ipilimumab $10 \mathrm{mg} / \mathrm{kg}$ every 3 weeks for 4 doses, with evaluation of response after 11 weeks of therapy. Patients who had evidence of clinical benefit then received maintenance therapy every 3 months with ipilimumab $10 \mathrm{mg} / \mathrm{kg}$. Dosing of ipilimumab was held for immune-related toxicities according to protocol guidelines.

Between March and May 2008, 3 patients developed grade 2 diarrhea typical of ipilimumab-induced colitis and were treated with infliximab $5 \mathrm{mg} / \mathrm{kg}$, mesalamine $800 \mathrm{mg}$ t.i.d., and loperamide. Although 1 patient responded promptly to this therapy, the other 2 patients had progressive colitis, requiring hospitalization for 13-15 days for intravenous (i.v.) steroids and total parenteral nutrition, in addition to the infliximab and mesalamine. Both recovered fully without complications. Our treatment for grade 2 diarrhea was amended in May 2008 to add daily hydrocortisone enemas, in addition to infliximab and mesalamine for the next 3 patients. Their case histories follow.

\section{Case histories}

Case 1. Mr LF is a 57-year-old man with desmoplastic melanoma who developed pulmonary metastases in 2007. Treatment with biochemotherapy in June 2007 produced a partial remission, but by January 2008, he had progressive disease. He was treated on the expanded access trial of ipilimumab receiving $10 \mathrm{mg} / \mathrm{kg}$ on February 14, March 16, and March 27, 2008. On April 17, 2008, he presented with hypotension and was found to have pituitary insufficiency with low plasma cortisol of $1.0 \mathrm{ug} / \mathrm{dL}$ and low plasma adrenocorticotrophin $(\mathrm{ACTH})$ of $10 \mathrm{pg} / \mathrm{mL}$. His hypotension responded promptly to $2 \mathrm{~L}$ normal saline and dexamethasone $4 \mathrm{mg}$ i.v., and he was begun on maintenance hydrocortisone $20 \mathrm{mg}$ in the morning and $10 \mathrm{mg}$ every evening. The following week, he had the onset of mild, grade 1 diarrhea, and on May 1, he was begun on mesalamine $800 \mathrm{mg}$ p.o. t.i.d., loperamide, and a low-fiber diet. Despite these measures, by May 15, the diarrhea had progressed to grade 2 with up to 10 watery stools daily with nausea, hematochezia, and 


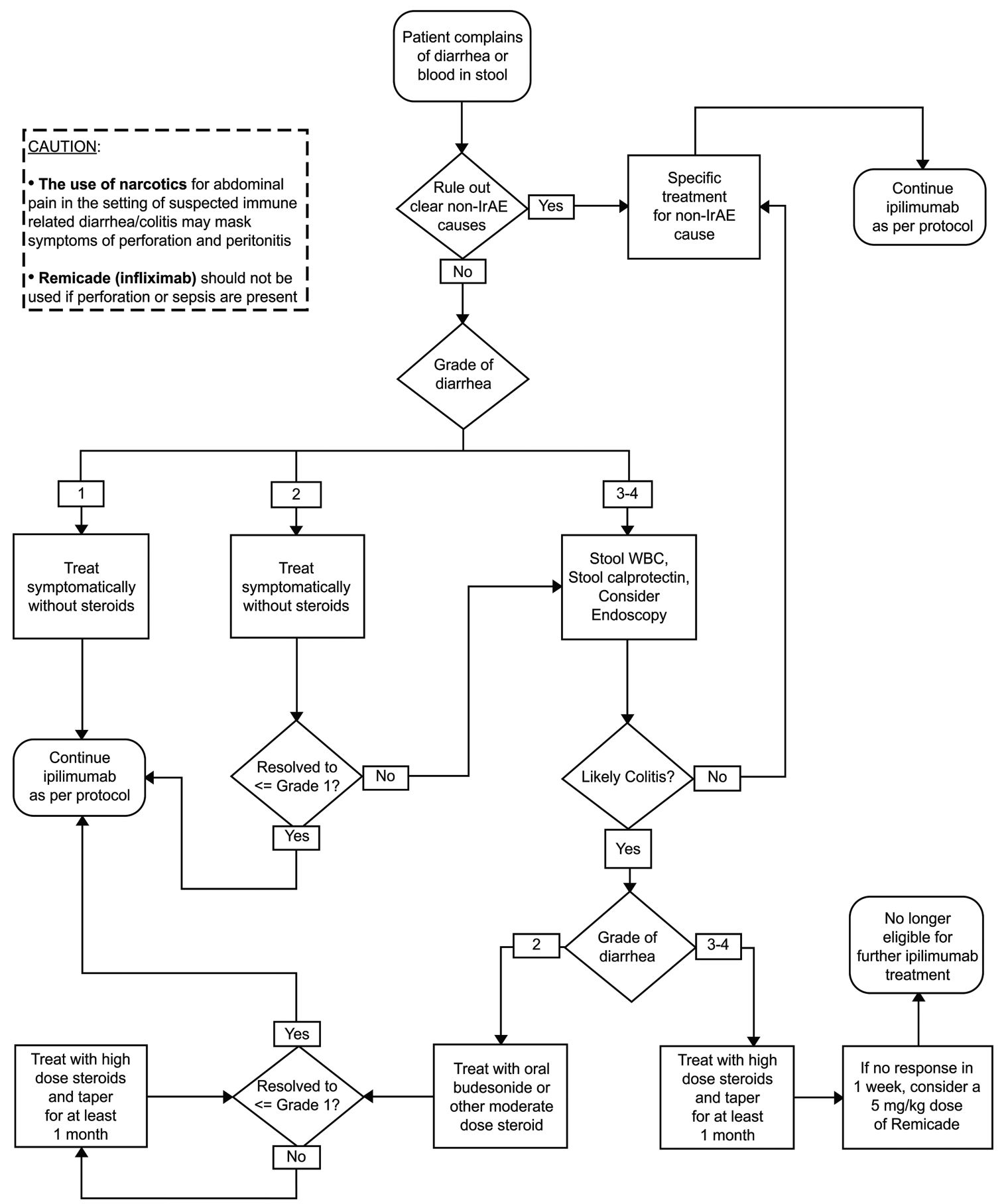

FIG. 1. The guidelines call for the close monitoring of the emergence or any change in symptoms and prompt treatment of ipilimumab-related diarrhea with corticosteroids. The algorithm provides recommendations for the treatment of diarrhea based on NCI-CTC grade. IrAE, immune-related adverse event; WBC, white blood cell.

dehydration with a 4-lb weight loss. He was treated as an outpatient with hydrocortisone $10 \%$ enemas once-daily and infliximab $5 \mathrm{mg} / \mathrm{kg}$ on May 16, and within a few days, his diarrhea had reduced to grade 1 with only 5 stools daily and normal hydration. He stopped the hydrocortisone enemas on May 30, continuing the mesalamine, but had some increase in diarrhea on June 26 that responded to a repeat 14-day course of hydrocortisone enemas and 1 more dose of in- fliximab on July 1 . His diarrhea was totally resolved by July 10 and has not recurred. Because of progressive melanoma his ipilimumab has been discontinued.

Case 2. Mr PR is a 47-year-old man who, after multiple therapies, developed subcutaneous (S.C.) and retroperitoneal metastases from melanoma in February 2008. He received 4 doses of ipilimumab $10 \mathrm{mg} / \mathrm{kg}$ between February 13 and 
April 18. Like the first patient, he developed cortisol deficiency in July with baseline cortisol $1.1 \mathrm{ug} / \mathrm{dL}$, low ACTH of $8 \mathrm{pg} / \mathrm{dL}$, and low poststimulation cortisol of $6 \mathrm{ug} / \mathrm{dL}$. His thyroid function was normal, and his fatigue resolved with replacement hydrocortisone. On July 25, he received his first maintenance dose of ipilimumab $10 \mathrm{mg} / \mathrm{kg}$. On August 8 , he had the abrupt onset of severe watery grade 2 diarrhea with hematochezia, nausea, and abdominal cramps, unresponsive to loperamide, with a weight loss of $6 \mathrm{lb}$ in 1 week. On August 11 , he received infliximab $5 \mathrm{mg} / \mathrm{kg}$ and began mesalamine $800 \mathrm{mg}$ p.o. t.i.d. and his replacement hydrocortisone was increased to $30 \mathrm{mg}$ in the morning and $15 \mathrm{mg}$ in the evening for 2 days. Within 2 days, his diarrhea had improved to grade 1 , and we added hydrocortisone enemas for 1 week, by which time the diarrhea had resolved.

Case 3. Mr TD is a 58-year-old man who presented in May 2006 with extensive metastatic disease involving many S.C., lymphatic, and GI sites complicated by GI bleeding and gram-negative septicemia. After an initial partial response to 6 cycles of biochemotherapy, by December 2006, he had progressive disease in all sites. He received 4 doses of ipilimumab between December 2006 and February 2007, with mild rash and vitiligo as his only side-effects. By November 2007, all measurable disease had regressed except one 12$\mathrm{mm}$ residual lesion in the perinephric fat. Ipilimumab therapy continued with maintenance doses of $10 \mathrm{mg} / \mathrm{kg}$ every 12 weeks, with his most recent dose on July 17, 2008. On August 6 , he had the onset of severe diarrhea with mild fever and rash and abdominal cramps, without improvement on loperamide and a liquid diet. When seen on August 18, his weight was down from 230 to $212 \mathrm{lb}$, and his leukocyte count was elevated at 14,500. In addition to grade 2 diarrhea, he had grade 1 rash and grade 1 conjunctivitis. He was treated as an outpatient with mesalamine $800 \mathrm{mg}$ t.i.d., hydrocortisone $10 \%$ enemas once-daily for 10 days, and infliximab $5 \mathrm{mg} / \mathrm{kg}$ on August 19 and September 2. His diarrhea improved to grade 1 by September 2 and was resolved by October 3.

\section{Discussion}

With the introduction of new immunologic agents into the treatment of cancer we see the appearance of new, unique toxicities. The ability to successfully treat these toxicities may be vital to the success of the new agent. IL-2 is an example of an immunologic agent where the toxicities remain problematic. The utility of IL-2 has always been hindered by a severe capillary leak syndrome that accompanies the administration of sufficient doses needed to achieve tumor regression.

Ipilimumab is an experimental therapy currently being investigated in patients with advanced melanoma and other malignancies. The safety profile of ipilimumab is characterized by irAEs that typically affect GI, skin, liver, and endocrine organs. For the most part, irAEs are mild to moderate and are either reversible or clinically manageable. In rare cases, $<1 \%$ irAEs may lead to serious complications. Diarrhea treatment guidelines have been developed and implemented in ipilimumab clinical trials (Fig. 1). An analysis of safety data comparing serious GI complications prior to and after full implementation of the diarrhea algorithm in- dicated that the treatment algorithm was effective in reducing the occurrence of serious GI complications, such as perforation or colectomy. ${ }^{8}$ Supported by extensive clinical experience utilizing the treatment algorithm in patients with ipilimumab-related diarrhea or colitis, systemic steroids are the first choice of treatment for severe or repetitive, moderategrade diarrhea or colitis. However, if patients do not respond to high-dose steroids within 1 week of therapy, consideration of infliximab therapy is appropriate according to the established guidelines.

The 3 patients with case histories reported in this article had grade 2 diarrhea (severe, watery diarrhea with dehydration lasting several days) that responded quickly to treatment with infliximab, mesalamine, and hydrocortisone enemas, allowing continued administration of immunotherapy with ipilimumab. Several reports illustrate the utility of continued administration of ipilimumab in maintaining a cancer remission or inducing a new remission. At our institution, we have observed that ipilimumab-induced grade 2 diarrhea was usually quickly followed by grade 3 diarrhea (requiring hospitalization), despite the institution of systemic corticosteroids, dietary modifications, and loperamide. This clinical experience led us to explore alternative approaches to the management of anti-CTLA-4-related grade 2 diarrhea or colitis.

\section{Conclusions}

The limitations of our study are apparent. Only 3 cases are reported, with short follow-up, and the favorable outcome of their treatment with infliximab may have been due to chance or other factors. We doubt that the mesalamine and hydrocortisone enemas alone were responsible for all the improvement we saw, as in our past experience these measures were not very successful. We did not perform colonic biopsies in these 3 cases, but we performed cultures to exclude infectious causes for the diarrhea, and the symptoms and clinical courses seemed typical of ipilimumab-induced colitis. We do not think the simultaneous pituitary deficiency in 2 patients affected the course of their colitis. During the same period, we saw several patients with ipilimumab-induced grade 1 diarrhea, a mild increase in stool frequency, and these patients responded to dietary modifications and loperamide, and we do not recommend infliximab for grade 1 diarrhea. We do not know whether the administration of infliximab will affect antitumor response rates or response duration. A recent study ${ }^{20}$ has demonstrated antitumor activity for infliximab when used for the treatment of renal-cell cancer, although direct antitumor activity is not expected in melanoma. We hope our brief report will stimulate further study of alternative therapeutic approaches to grade 2 diarrhea or colitis due to anti-CTLA4 monoclonal antibodies, in hopes of improving patient outcomes.

\section{Acknowledgments}

The authors wish to recognize and thank Kevin Man, M.D., for his advice and assistance in the care of the patients.

\section{Disclosure Statement}

David Minor has nothing to disclose. Kevin Chin is an employee of Bristol-Meyers Squibb. 


\section{References}

1. Weber J. Review of anti-CTLA antibody ipilimumab: Case studies of clinical response and immune-related toxicities. Oncologist 2007;12:864.

2. Kirkwood JM, Tarhini AA, Paneli MC, et al. Next generation of immunotherapy for melanoma. J Clin Oncol 2008;26:3445.

3. Urba WJ, Weber JS, O'Day DJ, et al. Long-term survival of patients with advanced melanoma who received ipilimumab administered at $10 \mathrm{mg} / \mathrm{kg}$ every 3 weeks for 4 doses (induction dosing). J Clin Oncol 2008;26(Suppl):7s.

4. Beck KE, Blansfield JA, Tran KQ, et al. Enterocolitis in patients with cancer after antibody blockade of cytotoxic Tlymphocyte-associated antigen 4. J Clin Oncol 2006;24:2283.

5. Yang JC, Hughes M, Kammula U, et al. Ipilimumab (antiCTLA4 antibody) causes regression of metastatic renal cell cancer associated with enteritis and hypophysitis. J Immunother 2007;30:825.

6. Weber JS, Berman D, Siegel J, et al. Safety and efficacy of ipilimumab with or without prophylactic budesonide in treatment-naïve and previously treated patients with advanced melanoma. J Clin Oncol 2008;26(Suppl):7s.

7. Smith FO, Goff, SL, Klapper JA, et al. Risk of bowel perforation in patients receiving interleukin-2 after therapy with antiCTLA 4 monoclonal antibody. J Immunother 2007;30:130.

8. Lin R, Yellin MJ, Lowy I, et al. An analysis of the effectiveness of specific guidelines for the management of ipilimumabmediated diarrhea/colitis: Prevention of intestinal perforation and/or colectomy. J Clin Oncol 2008;26(Suppl)7s.

9. Beer TM, Slovin SF, Higano CS, et al. Phase 1 trial of ipilimumab (IPI) alone and in combination with radiotherapy (XRT) in patients with metastatic castration resistant prostate cancer (mCRPC). J Clin Oncol 2008;26(Suppl):7s.

10. Tracey D, Klareskog L, Sasso EH, et al. Tumor necrosis factor antagonist mechanisms of action: A comprehensive review. Pharmacol Ther 2008;117:244.
11. Fries W, Muja C, Crisafulli C, et al. Dynamics of enterocyte tight junctions: Effect of experimental colitis and two different anti-TNF strategies. Am J Physiol Gastrointest Liver Physiol 2008;294:G938.

12. Oble DA, Mino-Kenudson M, Goldsmith J, et al. AlphaCTLA-4 mAb associated panenteritis: A histologic and immunohistochemical analysis. Am J Surg Path 2008;32:1130.

13. Berman D, Parker SM, Chasalow SD, et al. Potential immune biomarkers of gastrointestinal toxicities and efficacy in patients with advanced melanoma treated with ipilimumab with or without prophylactic budesonide. J Clin Oncol 2008; 26(Suppl):7s.

14. Lichenstein GR, Feagan BG, Cohen RD, et al. Serious infections and mortality in association with therapies for Crohn's disease: TREAT registry. Clin Gastroenterol Hepatol 2006;4:621.

15. Lin J, Ziring D, Desai S, et al. TNFa blockade in human diseases: An overview of efficacy and safety. Clin Immunol 2008;126:3.

16. D'Haens G, Baert F, van Assche G, et al. Early combined immunosuppression or conventional management in patients with newly diagnosed Crohn's disease: An open, randomized trial. Lancet 2008;371:660.

17. Caviglia R, Ribolsi M, Rizzi M, et al. Maintenance of remission with infliximab in inflammatory bowel disease: Efficacy and safety long-term follow-up. World J Gastroenterol 2007;13:5238.

18. Rutgeerts P, Sandborn WJ, Feagan BG, et al. Infliximab for induction and maintenance therapy for ulcerative colitis. NEJM 2005;353:2462.

19. Poggioli G, Laurenti S, Campieri M, et al. Infliximab in the treatment of Crohn's disease. Ther Clin Risk Manag 2007;3:301.

20. Harrison ML, Obermueller E, Maisey NR, et al. Tumor necrosis factor-alpha as a new target for renal cell carcinoma: Two sequential phase II trials of infliximab at standard and high dose. J Clin Oncol 2007;25:4542. 
